This item was submitted to Loughborough's Research Repository by the author.

Items in Figshare are protected by copyright, with all rights reserved, unless otherwise indicated.

\title{
Understanding human decision-making during production ramp-up using natural language processing
}

PLEASE CITE THE PUBLISHED VERSION

https://doi.org/10.1109/INDIN41052.2019.8972033

PUBLISHER

(C) IEEE

VERSION

AM (Accepted Manuscript)

\section{PUBLISHER STATEMENT}

(C) 2019 IEEE. Personal use of this material is permitted. Permission from IEEE must be obtained for all other uses, in any current or future media, including reprinting/republishing this material for advertising or promotional purposes, creating new collective works, for resale or redistribution to servers or lists, or reuse of any copyrighted component of this work in other works.

\section{LICENCE}

All Rights Reserved

\section{REPOSITORY RECORD}

Zimmer, Melanie, Ali Al-Yacoub, Pedro Ferreira, and Niels Lohse. 2020. "Understanding Human Decisionmaking During Production Ramp-up Using Natural Language Processing”. figshare. https://hdl.handle.net/2134/37930. 


\title{
Understanding Human Decision-making during Production Ramp-up using Natural Language Processing
}

\author{
Melanie Zimmer, Ali Al-Yacoub, Pedro Ferreira, Niels Lohse \\ Wolfson School of Mechanical, Electrical and Manufacturing Engineering \\ Intelligent Automation Centre \\ Loughborough University \\ Loughborough, LE11 3TU, UK \\ \{m.zimmer2, a.al-yacoub, p.ferreira, n.lohse\}@lboro.ac.uk
}

\begin{abstract}
Ramping up a manufacturing system from being just assembled to full-volume production capacity is a time consuming and error-prone task. The full behaviour of a system is difficult to predict in advance and disruptions that need to be resolved until the required performance targets are reached occur often. Information about the experienced faults and issues might be recorded, but usually, no record of decisions concerning necessary physical and process adjustments are kept. Having these data could help to uncover significant insights into the ramp-up process that could reduce the effort needed to bring the system to its mandatory state. This paper proposes Natural Language Processing (NLP) to interpret human operator comments collected during ramp-up. Recurring patterns in their feedback could be used to gain a deeper understanding of the cause and effect relationship between the system state and the corrective action that an operator applied. A manual dispensing experiment was conducted where human assessments in form of unstructured freeform text were gathered. These data have been used as an input for initial NLP analysis and preliminary results using the NLTK library are presented. Outcomes show first insights into the topics participants considered and lead to valuable knowledge to learn from this experience for the future.
\end{abstract}

Keywords- Industry 4.0; Natural Language Processing; Production Ramp-up; Unstructured Data; Decision-Support System.

\section{INTRODUCTION}

Ever shorter product life cycles and increasing customisation of products have become critical aspects for manufacturers in terms of their competitive advantage on the market. Rapidly assembling a manufacturing system and bringing it from low rate production to full capacity utilisation happens during the socalled production ramp-up [1]. Ramp-up is a time-consuming activity and often poorly understood [1]. This leads to a high likelihood of failure in relation to planned yield and duration [2]. Over the years, manufacturers have come to realise that in order to accomplish successful production ramp-ups, technology and human need to be better integrated during that phase [3] given the major dependence on a human to undertake changes to the system [4]. An increasing level of automation and end-to-end digitisation of the manufacturing domain, commonly referred to as Industry 4.0, supports this requirement. As more and more data become available, they can be used to aid shop-floor personnel in monitoring the ramp-up progress in real time [3]. This can enable a thorough understanding of underlying manufacturing processes, which is especially useful for the ramp-up to build and reuse knowledge in future ramp-up cases [3].

A vast number of auxiliary documentation created by technical staff, contain information about repair and quality issues and are commonly held in databases, written documents or files [5]. It has been estimated by Gartner that about $80 \%$ of organisational information assets are unstructured [6]. These unstructured data, however, resemble an important corporate resource owing to the detail that points to problems in design, production or deployment. In this context, the research work seeks to ultimately provide a decision-support framework for the ramp-up process in which recommendations will be offered to a human to facilitate the choice of change actions. As a first step towards this framework, a better comprehension of decisions that were made during the ramp-up process is required. Therefore, this research puts forward to dissect textual descriptions of manufacturing systems, processes and equipment gathered during the ramp-up phase using Natural Language Processing (NLP) tools. NLP can be described as "the application of automated parsing and machine learning techniques to analyse standard text" [7, p. 93]. As part of the presented work in this paper, this research will investigate what type of meaningful information can be extracted from natural language assessments acquired during a manual dispensing task.

\section{RELATED WORK}

To better appreciate the significance of ramp-up for the manufacturing process, it is essential to understand the characteristics and difficulties experienced throughout that phase. The duration of ramp-up is not straightforward to specify, but it can be understood as the period from once the system is first put together and prototype production has started until the specified performance targets are achieved [8]. Key performance indicators for ramp-up are usually production quality [9], [10] and yield [1]. These however highly fluctuate during ramp-up as various unexpected malfunctions can occur, which can be costly and time-consuming [8]. Disturbances usually arise due to the non-repetitiveness of the ramp-up that increases the complexity and unpredictability of the process [11], [12]. This uniqueness of each ramp-up scenario as well as the necessary process and equipment adjustments make it necessary to rely on the expertise and intervention of a human [13]. This often leads to trial-and-error decisions based on the operator's judgement and can vary extremely among different 
people. In order to be able to draw from the benefits that Industry 4.0 can bring, the availability of data is vital. However, especially when it comes to ramp-up, production and historical data are usually not available and design information can even be incorrect [3].

Increasing automation and self-learning capabilities are a big aspect of Industry 4.0. Studies show [14] that when asked about the function of the human in the ramp-up of the future, high significance is given to the aspect of problem-solving. Machines, on the other hand, are said to play an important role during ramp-up management. These studies furthermore suggest that operators will have their personal machine assistant to support them in the decision-making process, but the ultimate decision remains with the human. Previously, research has looked at different ways of preserving the operator's knowledge during ramp-up [4], [15], [16]. Up to now, there is still a need to facilitate the reuse of this knowledge as manual evaluation and classification of it is not only time consuming, but similarly labour intensive. One field that is concerned with making a computer understand natural language by exploring constructs of a text semantically and grammatically is NLP [17]. Nowadays, NLP is widely used in social media [18], but other applications can also be found in the manufacturing domain. These include areas such as work instructions [19] and automated requirements specification analysis [20] or product quality and reliability improvements based on customer data [21]. Although the use of natural language is intuitive for the human, this usually causes problems to a machine and can complicate further analysis. This results from language usually differing from person to person and different meanings of words depending on the context they are used in [20]. This not only leads to inconsistency but also inaccuracy and ambiguity [22].

Before text can automatically be analysed and features extracted, several pre-processing steps are required [17]. Nowadays, there are a variety of NLP libraries available that can help with these tasks. Among the commonly used toolkits are Natural Language Toolkit (NLTK), Apache OpenNLP and Stanford CoreNLP [23].

\section{PROBLEM DEFINITION}

Existing ramp-up solutions are generally human-in-loop systems with the human operator being the primary resource of knowledge. The decisions that are made during ramp-up are reliant on a human to be carried out. Although standard procedures are not available, the current ramp-up process follows a certain cycle that is typical for it: After the operator has put the individual elements (processes and equipment modules) of the system in place for the first time, a test run will be conducted. This test run will yield several results in the form of the product, key performance indicators (KPIs) of the system as well as the system itself. The operator inspects these different outcomes based on existing knowledge or given target KPIs. After an adjustment has been made and the assessment has been captured, another test run will follow. This again will result in the entities identified above and the process of assessing and adjusting continue.

Based on this simplified ramp-up explanation, it can be seen that data about the system state in form of process and equipment settings, current and target KPIs, as well as human input, would be useful to know about the ramp-up. The capture and availability of this in combination with the aforementioned data create an opportunity to form more knowledgeable decisions as the interconnected system data can be linked with the human input to improve the analysis of task-specific approaches as appearing in ramp-up. Current production data are needed to serve as an input to real-time decision support. Thus, a representation of the manufacturing system as a cyber-physical system is desirable so that data are accessible and can be stored in a cloud system. In that regard, the problem that is targeted in this research is the extraction of expert knowledge of adjustment actions, product quality and performance indicators.

Although initial research has already been undertaken to structure and capture these data, additional analysis of free-form text is still lacking. NLP has been applied to written human language in manufacturing to help understand the structure and meaning of that text. But, to the best of the authors' knowledge, it has not been applied to the ramp-up case. Yet, this seems a suitable choice to extract structured information from loosely collected text data inputs during ramp-up. Analysing human input about incorrect physical settings, faulty processes or any other disruptions will lead to a valuable comprehension of knowing what went wrong in the past that can be prevented in the future. More importantly, the identification of solutions that have been deployed by shop-floor personnel plays a crucial role in improving the ramp-up process. This will allow to not only reuse and share the information but what is more, create a knowledge base over time. An illustrative overview of the current and anticipated ramp-up process is shown in Fig. 1.

The main challenges that will have to be addressed in this research are identified as follows: First, as this research focusses on examining the operators' knowledge, the main input into the analysis will be human written language. This language is often imperfect, and ambiguities may exist. In addition, as the focus is on ramp-up specific cases, the use of technical jargon applied by the shop-floor personnel is to be expected. Lastly, complex data relationships occur that link not only across different ramp-up scenarios but moreover between different operators.

\section{METHODOLOGY}

The overall intention of this work-in-progress is to develop a recommendation system in the form of a question-and-answer mechanism leveraging previously built knowledge that can help the user by offering advice for setup actions. The scope of this paper covers the investigation of whether meaningful information can be extracted from sparse and unstructured data inputs acquired during the ramp-up process. It is therefore proposed as part of this research to use NLP practices to study the benefit of NLP for the ramp-up process in the context of identifying significant information that can be extracted from operator assessments. As a first step, primary text analyses are conducted using an NLP library to obtain initial insights. The selection criteria for choosing a suitable library have been defined as follows: (1) support of English language, (2) is opensource software, (3) provides wrappers for Python, which facilitate the integration into the existing experimental setup, (4) offers comprehensive documentation, and (5) allows for a certain degree of adaptation to choose amongst different 
algorithms. Fig. 1 illustrates how the presented method will fit in the current situation of the ramp-up process.

To facilitate the capabilities of NLP, firstly human knowledge needs to be collected while carrying out a ramp-up task. As an input, a CSV file containing the assessments inputted in human language will be used. But before the data can be further processed, they need to be cleaned to begin with. Raw free text format is not optimal as it might contain unnecessary empty spaces, lines or even rows, which will have to be removed. In some cases, it might on top of that be necessary to remove whitespaces between two consecutive words, e.g. between a number and a unit. Hence, it is essential to filter out all these problems prior to the processing stage. In the context of this research, the following NLP processing steps [7], [18] are considered for obtaining structured entities from the unstructured text:

1) Tokenisation: Process of splitting the input into smaller pieces. A token can, in this matter, be a single word, multiple words or punctuation.

2) Same case: Converting input to upper or lower case to treat variations of the word the same, if this distinction is not vital.

3) Removal of stop word and punctuations: Stop words add little to the overall meaning and typically occur most frequently in a language, such as articles and conjunctions.

4) Stemming and lemmatisation: Stemming is the removal of affixes of a word to find the root, for instance, "swimming" becomes "swim". Although similar, lemmatisation provides the dictionary form of a word, such as "wolves" to "wolf". This process is usually more time consuming than stemming. As the outputs will be mandatory to be fed back to a human, it is crucial that the structured data will be still understandable and useable.
5) Part-of-speech (POS) tagging: The usage of POS tagging allows to identify whether a token is a noun, an adjective, a verb, a numeral, etc. within the given sentence. This can be quite a difficult task as a word can represent a different part depending on the context, such as the word "move".

6) Shallow parsing or chunking: Analysis of a sentence to link identified parts of sentences like nouns, verbs, adjectives, etc. to higher order entities that have a grammatical meaning, such as a group of nouns, phrases or verbs, etc.

7) N-grams: N-grams are used for the prediction of the next most likely word. They depict a group of adjacent characters or words consisting of n-tuples. The sentence "This is a sentence" can, for example, be divided into unigrams ("this", "is", "a", "sentence"), bigrams ("this is", "is a", "a sentence") or trigrams ("this is a", "is a sentence").

8) Term frequency-inverse document frequency (TF-IDF): TF-IDF is a statistical measure to indicate the importance of a word in a document corpus. The importance increases proportionally to the number of times a word appears in the document but is offset by the frequency of the word in the corpus. TF-IDF is calculated as:

$$
t f i d f(t, d, D)=t f(t, d) \cdot i d f(t, D),
$$

Where $t f(t, d)$ is equal to $\frac{f_{t, d}}{\sum_{t^{\prime} \in d} f_{t^{\prime}, d}}$ the times term $t$ appears in a document $d$ divided by the total number of terms included in document $d$, whereas $\operatorname{idf}(t, D)$ is equal to $\log \frac{N}{|\{d \in D: t \in d\}|}$, for which $N=|D|$ is the total number of documents and $\mid\{d \in$ $D: t \in d\} \mid$ is the number of documents that contain term $t$.

9) K-means clustering: Unsupervised learning algorithm that divides a set of data points into natural grouping(s) so that points inside one cluster are similar to one another. The

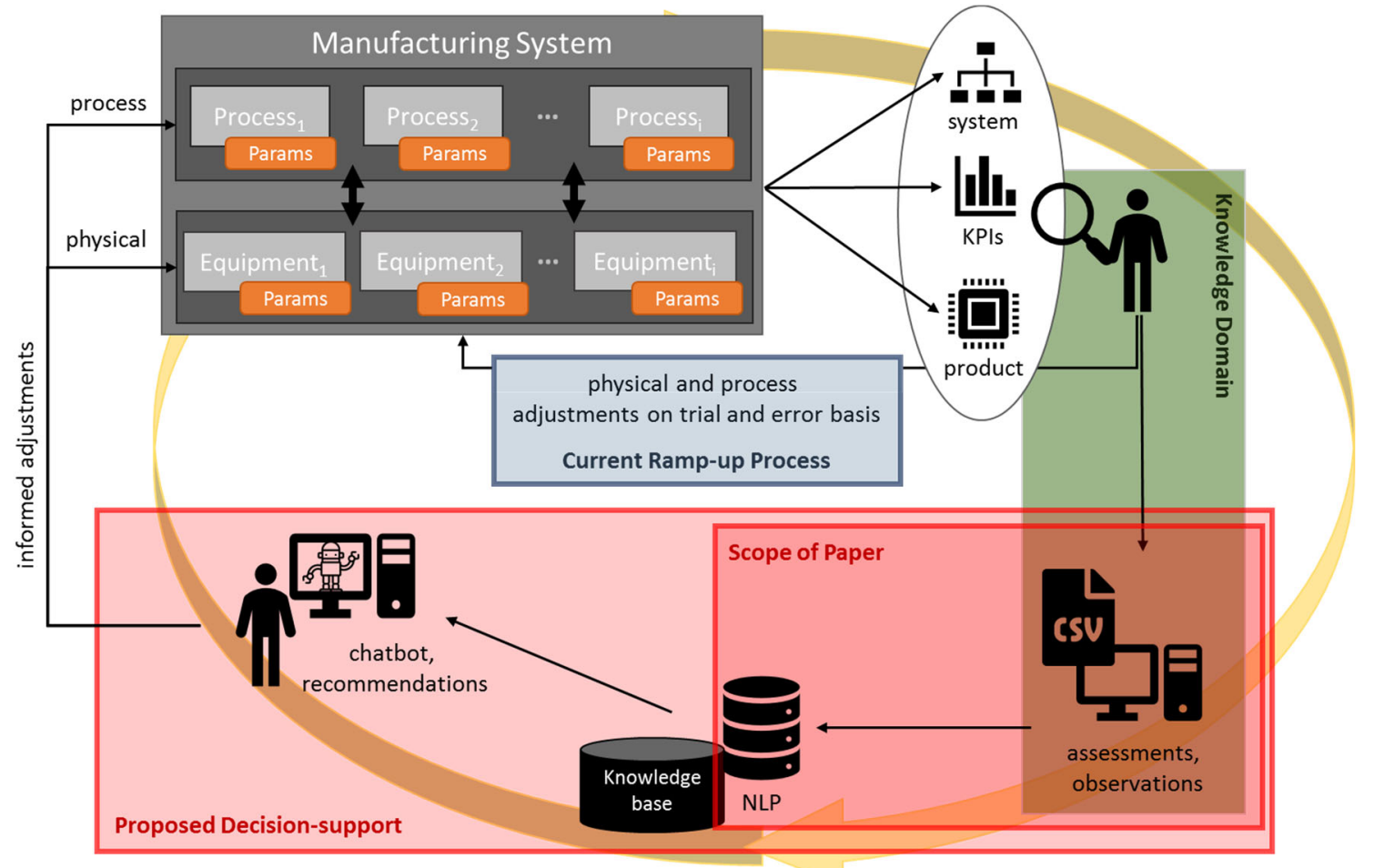

Fig. 1. Overview of overall methodology in context of the ramp-up process and scope of paper. 
algorithm will be smartly initialised with a fixed number $k$ of socalled cluster centroids. It is an iterative approach and assigns each data point to the nearest cluster, followed by moving the centroid based on the average of the points contained in the cluster. Although other clustering algorithms might reveal better results, for the purpose of this research k-means has been chosen as a first starting point due to its speed and simplicity [24].

By doing the above, it is envisioned to lay the basis for work in the area of decision-making for the operator during the rampup process as it is believed that expert knowledge can be extracted to reveal useful insights for future ramp-up processes to the user.

\section{CASE Study}

A manual dispensing experiment was conducted for an initial data collection (Fig. 2). Each of the twenty participants was asked to produce several patterns during this ethics-approved experiment ${ }^{1}$. For the setup, a Schunk gripper with 3D-printed handle was controlled by the participants. A push button was attached to the gripper and connected to a time-pressure dispensing unit (Fisnar JB1113N). This allowed triggering the process by which toothpaste was released through a syringe. Participants could make certain adjustments to the setting. Besides changing the nozzle diameter of the syringe between $1.2 \mathrm{~mm}$ and $1.6 \mathrm{~mm}$, the dispensing pressure could be chosen from the values 10, 16 and 20 psi. The speed of the gripper was in this setup directly controlled by the participant through the hand movement.

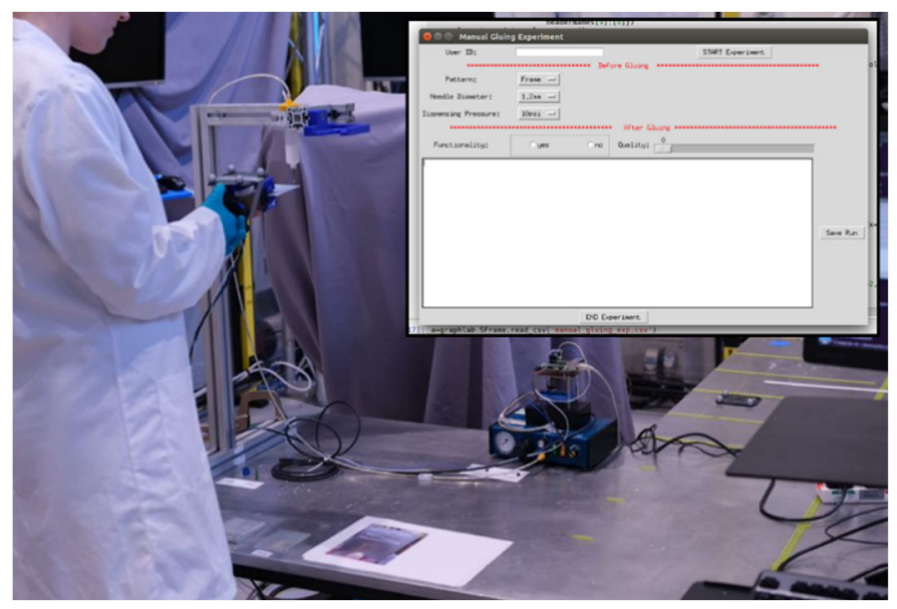

Fig. 2. Participant during the manual dispensing task. The developed graphical user interface to capture the assessment is seen at the top righthand corner.

With the intention of being able to replicate good decisions in the future, participants were asked to assess the equipment functionality, the process itself, as well as the produced pattern through a graphical user interface in form of radio buttons, freeform text and a score between 0 and 100. Good results were identified by continuous and straight lines as well as close similarity to the desired patterns (frame, raster or zig-zag). Sample outcomes are given in Fig. 3.

\footnotetext{
${ }^{1}$ Ethics Approvals (Human Participants) Sub-Committee at Loughborough University
}
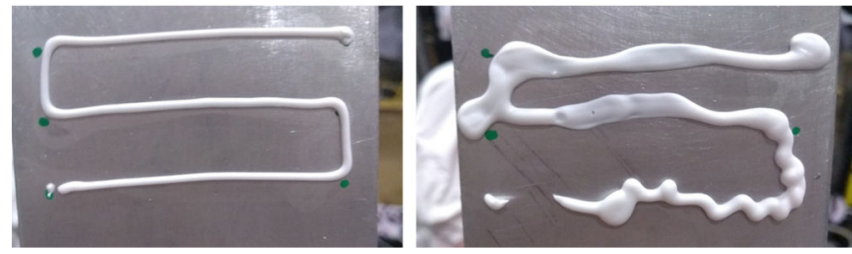

Fig. 3. Example outcomes of the dispensing task. The picture on the left shows a good example, whereas the right has discontinuous and uneven lines.

\section{RESULTS}

The preliminary results presented in the following subsections were achieved using NLTK (v3.4) [25], Python (v2.7.15) and scikit-learn (v0.20.2) on data from the manual dispensing experiment.

\section{A. Data Description}

A total of 232 usable records was collected during the experiment, with an average assessment text size of about 19 words. As was expected, an exploratory inspection of the text revealed differences between the participants' writing styles as some used short phrases ("not quite straight") instead of full sentences. Besides, some spelling mistakes ("squred", "patern", "tooth paster") and in rare cases colloquial language ("kinda") were found. After the data had been cleaned, data pre-processing as has been outlined in Section IV was performed.

\section{B. Text Syntax and Structure}

POS tagging allows to break the assessments down into specific parts, such as most frequently used nouns, adjectives or even verbs across the whole of the dataset. Plotting the first few nouns that have been used the most by the different participants (Fig. 4), uncovered that comments were often made on the parameters that could be changed during the experiment. These are for example the pressure or needle diameter.

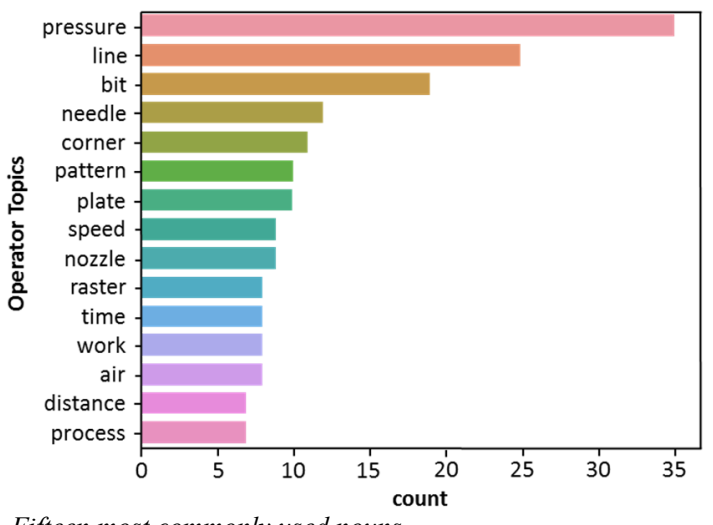

Fig. 4. Fifteen most commonly used nouns.

It is to be noted that 'nozzle' and 'needle' seem to have been used interchangeably and this will have to be taken into further consideration for future processing. Words such as 'line', 'corner' or 'plate' refer to comments made about the produced pattern. The word 'air' most likely relates to issues that have been recorded by the participants concerned air bubbles accumulating in the dispensing syringe ("Still air in the system 
there was an audible pop during dispensing."). The words 'bit' and 'work' seem to be most likely misidentified as nouns and it might be necessary to remove these words from the text.

Whereas an overview of commonly used nouns was quite insightful, making sense of the fifteen most commonly used adjectives is not so simple by just looking at the data alone (Fig. 5). Here, the most frequently used adjective is "good". This could relate to many things in the experimental setup or the produced result. In this case, it would be fundamental and interesting to consider other data that have been gathered about the process such as the setting and quality assessment. Words such as 'difficult' and 'easy' most likely refer to the dispensing process itself and 'last', 'first' as well as 'second' could relate to the different trials that have been conducted by each participant.

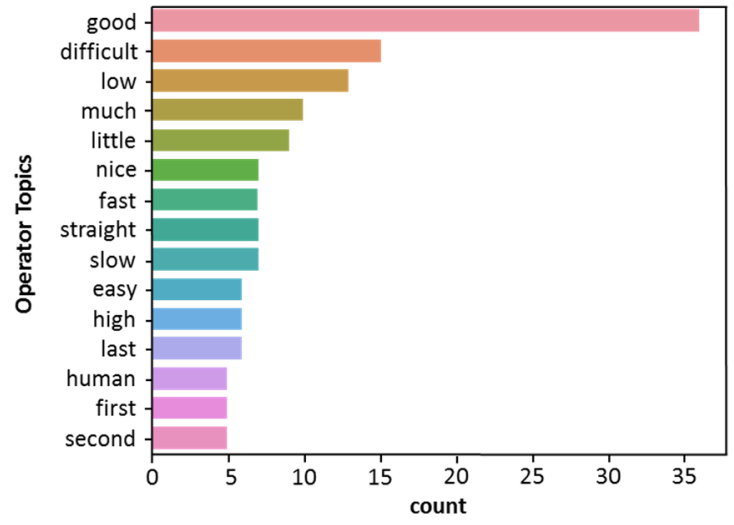

Fig. 5. Fifteen most commonly used adjectives.

\section{Feature Extraction}

Instead of examining words in isolation, the use of n-grams, where $n>1$, can be especially useful as they can capture which word is more likely to follow another one. The bigger the number $n$, i.e. the longer the n-gram, the more context is given. The ideal choice of $n$ is again dependent on the application as too small n-grams might not provide any detail, whereas ngrams that are too big might not generalise well. Different ngrams for $n=2, \ldots, 4$ have been created over all the collected assessments text using scikit-learn's TfidfVectorizer. For bigrams, 136 features were found, 21 for trigrams, and two for 4-grams. Sample outcomes for the different n-grams are presented in Table 1.

Table 1. Sample outcomes for bi-, tri-, and 4-grams.

\begin{tabular}{|l|l|}
\hline \multirow{4}{*}{ n-grams } & Sample features \\
\hline \multirow{4}{*}{ bigrams } & air bubbl \\
\cline { 2 - 2 } & pressur good \\
\cline { 2 - 2 } & seem perfect \\
\hline \multirow{3}{*}{ trigrams } & pressur drop unexpect \\
\cline { 2 - 2 } & materi stick needl \\
\cline { 2 - 2 } & seem work best \\
\hline \multirow{3}{*}{ 4-grams } & good nozzl diamet also \\
\cline { 2 - 2 } & pressur good nozzl diamet \\
\hline
\end{tabular}

These outcomes are very informative in the sense that they can already convey a good indication of what the participants experienced during the experiment as now more context is specified. Participants seem to have experienced issues with the dispensing material itself ("air bubbl") and in combination with the nozzle ("materi stick needl"). On the other hand, information about the equipment settings has been provided (e.g. "pressure good" and "good nozzl diamet also"). Interesting are also features such as "seem perfect" or "seem work best". This will especially be of relevance for future efforts, where a mapping of these features to the parameter settings will be considered.

\section{Text Clustering}

In addition to the previous step, scikit-learn's k-means clustering was applied and initialised using k-means ++ . Doing so allowed grouping the assessments into unique clusters that were then linked to the individual operator. As the k-means algorithm can be set up with varying parameters, the elbow method was used to determine a suitable number of initial clusters. The elbow method is run over several values of $k$ and for each run, the sum of squared distances of each data point to its nearest cluster centroids was considered. For the manual dispensing experiment data, an initialising value of 26 has been found to be tolerable. In spite of that, due to better readability, the algorithm was initialised with 12 clusters and the resulting clusters were then mapped to the operators in a heatmap as seen in Fig. 6. On the left side of the figure, the seven most frequent words from the respective clusters are given. On the right side, the generated heatmap is visualised with the user IDs on the $\mathrm{x}$ axis and cluster numbers on the y-axis. It can be seen that "op19" for instance used words appearing in cluster " 10 " more often than operator 18. Linking this information to the individual trials of these two operators, for example, will show the different physical and process settings that have been made in combination with the different ramp-up techniques that were applied. By doing this mapping, it is envisioned that this will possibly lead to an understanding of why operator 19 seemed to encounter problems with the corners and the release of the pushbutton, whereas operator 18 did not.

\section{CONCLUSION AND FUTURE WORK}

This paper presents the first step into gaining insight through the use of NLP to support a human during the ramp-up process. For this, NLTK has been chosen as a starting point for its ease of integration into an existing research environment and available functionalities. The library was applied to data that were originally gathered in a manual dispensing experiment. During this experiment, participants were asked to assess the outcome of a produced pattern in the form of free-form texts and scores. While this work is not conclusive, preliminary data show that applying NLP to the available dataset did offer insights into topics and problems that have been encountered mostly by the participants during the experiment. In particular n-grams, TFIDF and k-means are interesting approaches to this as they allow to get more information through context. These steps are however just the beginning and there is increased potential in other aspects of NLP. More advanced NLP methods such as sentiment analysis, which is often used in a social media context, will be further investigated. This will be done to establish whether a correlation between the assigned production quality score and the sentiment of the operator's assessment correspond to another. This might require to not only focus on NLTK but also other NLP tools. Additionally, it will be investigated 


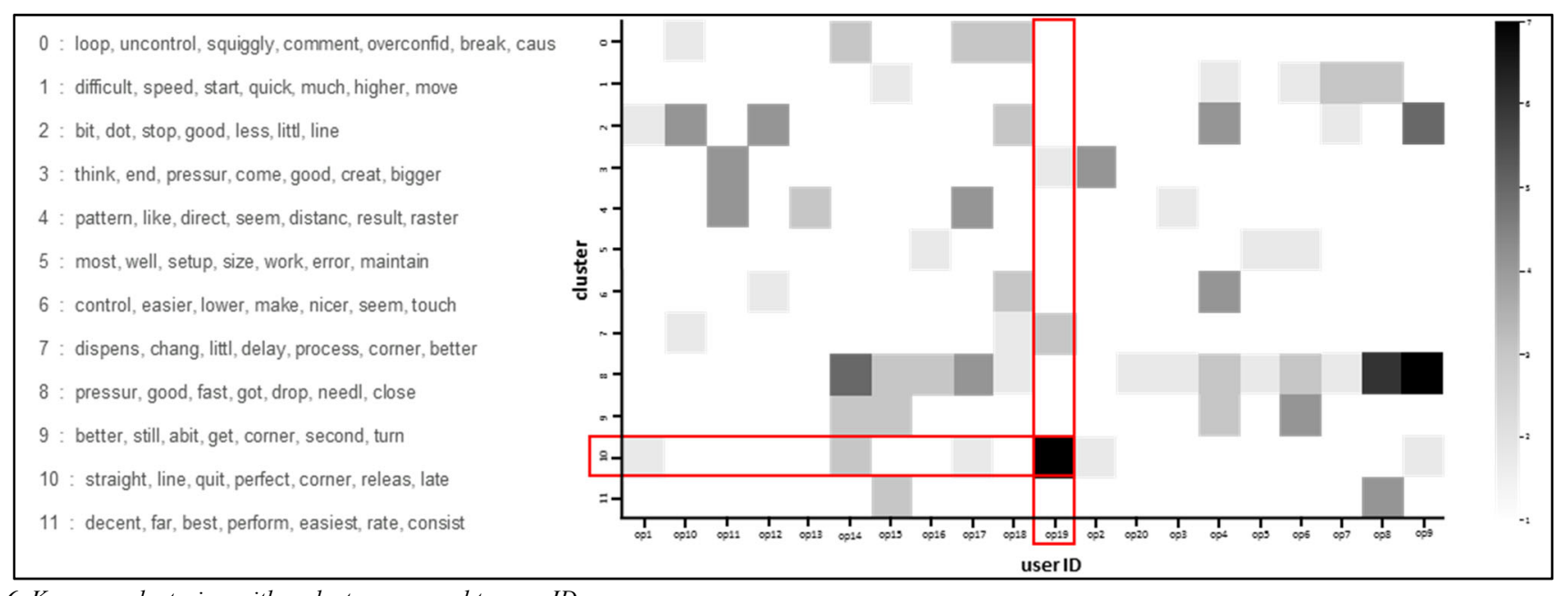

Fig. 6. K-means clustering with $n$ clusters mapped to user IDs.

whether the use of written or spoken comments has an impact on the assessments that the operators provide. One aspect that is assumed is, that the detail of written comments decreases over time as this process could be deemed quite cumbersome. As a long-term goal, the development of a chatbot is foreseen that will help the operator through the decision-making of the ramp-up process by providing recommendations or additional considerations. Consequently, to have a larger data set with a link to quality and physical settings, this work will organise a subsequent experiment in the near future, where participants will be required to ramp-up a small-scale glueing workstation.

\section{ACKNOWLEDGEMENT}

The authors gratefully acknowledge the financial support of the Engineering and Physical Sciences Research Council (EPSRC) Centre for Doctoral Training in Embedded Intelligence under grant reference EP/L014998/1. The research leading to these results has also received funding from the European Union's Horizon 2020 research and innovation programme under grant agreement No 680735, project openMOS (Open Dynamic Manufacturing Operating System for Smart Plug-and-Produce Automation Components).

\section{REFERENCES}

[1] C. Terwiesch and R. E. Bohn, "Learning and process improvement during production ramp-up," Int. J. Prod. Econ., vol. 70, pp. 1-19, 2001.

[2] M. Slamanig and H. Winkler, "Management of product change projects: a supply chain perspective," Int. J. Serv. Oper. Manag., vol. 11, no. 4, p. 481, 2012.

[3] U. Dombrowski, J. Wullbrandt, and P. Krenkel, "Industrie 4.0 in production ramp-up management," in Procedia Manufacturing, 2018.

[4] D. Scrimieri, R. F. Oates, and S. M. Ratchev, "Learning and reuse of engineering ramp-up strategies for modular assembly systems," J. Intell. Manuf., vol. 26, no. 6, pp. 1063-1076, 2015.

[5] M. M. Kornfein and H. Goldfarb, "A Comparison of Classification Techniques for Technical Text Passages," in Proceedings of the World Congress on Engineering 2007, 2007.

[6] D. Stewart, "Big Content: The Unstructured Side of Big Data," Gartner Blog Network, 2013. [Online]. Available: https://blogs.gartner.com/darinstewart/2013/05/01/big-content-the-unstructured-side-of-big-data/. [Accessed: 04-Mar-2019].

[7] N. Lindblad et al., "Natural Language Processing of Textual Requirements," ICONS 2015 Tenth Int. Conf. Syst. Nat., 2015.

[8] M. Colledani, T. Tolio, and A. Yemane, "Production quality improvement during manufacturing systems ramp-up," 2018.
[9] J. Fleischer, D. Spath, and G. Lanza, "Quality simulation for fast ramp up," in Proceedings of the 36th CIRP International Seminar on Manufacturing Systems, 2003.

[10] S. Fjällström, K. Säfsten, U. Harlin, and J. Stahre, "Information enabling production ramp-up," J. Manuf. Technol. Manag., vol. 20, no. 2, pp. 178196, 2009.

[11] S. C. Doltsinis, S. Ratchev, and N. Lohse, "A framework for performance measurement during production ramp-up of assembly stations," Eur. J. Oper. Res., vol. 229, no. 1, pp. 85-94, 2013.

[12] G. Schuh, T. Gartzen, and J. Wagner, "Complexity-oriented ramp-up of assembly systems," CIRP J. Manuf. Sci. Technol., vol. 10, pp. 1-15, 2015.

[13] S. Doltsinis, P. Ferreira, and N. Lohse, "Reinforcement learning for production ramp-up: A Q-batch learning approach," Proc. - 2012 11th Int. Conf. Mach. Learn. Appl. ICMLA 2012, vol. 1, pp. 610-615, 2012.

[14] R. Schmitt et al., "On the future of ramp-up management," CIRP $J$. Manuf. Sci. Technol., vol. 23, pp. 217-225, 2018.

[15] “"open Dynamic Manufacturing Operating System for Smart "Plug-andProduce" automation components' (openMOS)." [Online]. Available: www.openmos.eu.

[16] K. Konrad, M. Hoffmeister, M. Zapp, A. Verl, and J. Busse, "Enabling fast ramp-up of assembly lines through context-mapping of implicit operator knowledge and machine-derived data," in IFIP Advances in Information and Communication Technology, 2012.

[17] F. Zhang, H. Fleyeh, X. Wang, and M. Lu, "Construction site accident analysis using text mining and natural language processing techniques," Autom. Constr., vol. 99, pp. 238-248, Mar. 2019.

[18] A. Pinto, H. Gonçalo Oliveira, and A. Oliveira Alves, "Comparing the Performance of Different NLP Toolkits in Formal and Social Media Text," in 5th Symposium on Languages, Applications and Technologies (SLATE'16), 2016.

[19] R. S. Renu and G. Mocko, "Text Analysis of Assembly Work Instructions," in International Design Engineering Technical Conferences \& Computers and Information in Engineering Conference IDETC/CIE 2015, 2015.

[20] W. M. Wilson, L. H. Rosenberg, and L. E. Hyatt, "Automated analysis of requirement specifications," in Proceedings - International Conference on Software Engineering, 1997.

[21] A. Brombacher et al., "Improving product quality and reliability with customer experience data," Qual. Reliab. Eng. Int., 2012.

[22] M. Bates and R. M. Weischedel, Challenges in natural language processing. Cambridge University Press, 1993.

[23] S. Sun, C. Luo, and J. Chen, "A review of natural language processing techniques for opinion mining systems," Inf. Fusion, Jul. 2017.

[24] A. K. Jain, "Data clustering: 50 years beyond K-means," Pattern Recognit. Lett., 2010.

[25] S. Bird, E. Loper, and E. Klein, Natural Language Processing with Python. O’Reilly Media Inc., 2009. 